\title{
THE DETERMINATION OF A PARABOLIC EQUATION FROM INITIAL AND FINAL DATA
}

\author{
WILLIAM RUNDELL
}

\begin{abstract}
It is shown that an unknown, spatially-dependent coefficient $a(x)$ in the parabolic equation $u_{t}-\Delta u+a(x) u=0$ can be determined from a knowledge of both initial and final data. An existence and uniqueness theorem is given and the continuous dependence of the function $a(x)$ on the data is examined.
\end{abstract}

1. Introduction. The problem of trying to find the unknown coefficient $a(x)$ in the parabolic equation

$$
u_{t}-u_{x x}+a(x) u=0
$$

has received considerable recent interest. Typically one gives primary boundary conditions with which one could uniquely determine $u(x, t)$ if $a(x)$ were known, for example the initial-boundary data $u(0, t), u(1, t)$, and $u(x, 0)$. One then gives additional boundary conditions in order to attempt to determine $a(x)$, and this has often been the flux on one of the lines $x=0$ or $x=1$, for example $u_{x}(0, t)$. In [4] Pierce showed that if $u(x, 0)=0, u(1, t)=0$, and $u(0, t), u_{x}(0, t)$ are prescribed, then there is at most one solution pair $(a(x), u(x, t))$. For the case $u(0, t)=u(1, t)=0$ and $u(x, 0), u_{x}(0, t)$ given he was able to show uniqueness only if the initial value $u(x, 0)$ had nonvanishing Fourier coefficients with respect to the basis generated by $\phi_{n}(x)$, the eigenfunctions for the Sturm-Liouville operator

$$
-\phi+a(x) \phi_{n}=\lambda_{n} \rho_{n}, \quad \phi_{n}(0)=\phi_{n}(1)=0 .
$$

The method used-indeed the method that forms the basis for most of the approaches to this problem-is to convert it to an inverse Sturm-Liouville problem and then use the techniques developed by Gel'fand and Levitan [3]. In a series of papers, for example [7], Suzuki has considered the problem of giving Cauchy data on both $x=0$ and $x=1$ and has shown that one can uniquely recover both $a(x)$ and the initial value $u(x, 0)$. Again the approach is via the Gel'fand-Levitan method for the inverse Sturm-Liouville problem.

The determination of $a(x)$ under these conditions does not lead to a well-posed problem, since $a(x)$ does not depend continuously on the initial-boundary data in what would be considered the usual norms [5].

The author, in a different attack [6], constructed an integral operator that mapped solutions of

$$
u_{t}-u_{x x}+a(x) u=0
$$

onto solutions of

$$
v_{t}-v_{x x}+b(x) v=0
$$

Received by the editors September 20, 1985.

1980 Mathematics Subject Classification (1985 Revision). Primary 35R30, 35K15. 
where $u$ and $v$ share the same Cauchy data on $x=0$. If in addition $u(x, 0)=v(x, 0)$ for $x>0$, then $a(x)=b(x)$ for $x>0$. This approach uses the Cauchy data on $x=0$ as the primary boundary conditions and the initial value $u(x, 0)$ as the additional condition that allows the determination of $a(x)$. This formulation has the advantage of not directly referencing the eigenfunctions of the Sturm-Liouville operators, but it does not lead to a well-posed problem since the Cauchy problem for (1.3) is illposed and one does not get continuous dependence of $a(x)$ on $u(0, t), u_{x}(0, t)$, and $u(x, 0)$ in the supremum norm.

For equation (1.1) in more than one space variable, attempts to recover $a(x)$ for $x \in \mathbf{R}^{n}$ with $n>1$ have proved fruitless. The Gel'fand-Levitan approach appears to be limited to ordinary differential equations; one of the main stumbling blocks to an extension to several variations is that the eigenfunctions $\phi$ satisfying

$$
-\Delta \phi+a(x) \phi=\lambda \phi
$$

no longer have single multiplicity. The integral operator method $[\mathbf{5}, \mathbf{6}]$ seems to be limited to one space variable for a variety of reasons.

This leads to two natural questions concerning the determination of an unknown $a(x)$. First what set of overposed boundary conditions can be used to determine both $a(x)$ and $u(x, t)$ in the equation

$$
u_{t}-\Delta u+a(x) u=0 ?
$$

Second, even in the case $n=1$ are there any conditions that lead to a well-posed problem for determining $a(x)$ ?

It is a "folk theorem" in undetermined coefficient problems that one usually gets a well-posed problem if the additional data is prescribed in a direction "parallel" to the coefficient but not if it is "perpendicular" [1]. Thus this would predict, as has been found, that to determine $a(x)$ from $u(0, t), u(1, t)$, and $u(x, 0)$ as primary initial-boundary conditions and imposing $u_{x}(0, t)$ as the additional condition would be ill-posed since we are attempting to obtain a function of $x$ by prescribing a function of $t$. In [5], where the "parallel" data $u(x, 0)$ was used as the extra condition, the problem was ill-posed because the primary problem, namely the Cauchy data $u(0, t)$ and $u_{x}(0, t)$, does not constitute a well-posed problem for (1.1).

Since the primary conditions $u(0, t), u(1, t)$, and $u(x, 0)$ yield a well-posed problem for $u(x, t)$ is there any additional condition in the $x$-direction that one can impose that would lead to a well-posed problem for $a(x)$ ? The obvious choice is to give $u(x, T)$ for some $T>0$. It is the purpose of this paper to show that uniqueness holds for this problem, that is there is at most one coefficient $a(x)$ in (1.6) that takes on prescribed values of $u(x, 0)$ and $u(x, T)$. An example is given to show that one cannot in general obtain continuous dependence on the boundary data unless the class of coefficients considered is suitably restricted.

2. Uniqueness. Let $\Omega$ be a bounded open subset of $\mathbf{R}^{n}$ whose boundary $\partial \Omega$ is an $n-1$ dimensional manifold lying on one side of $\Omega$. Let $\partial \Omega$ be of class $C^{2+\alpha}$, that is each point of $\partial \Omega$ has a neighborhood in which $\partial \Omega$ is the graph of a $C^{2+\alpha}$ function of $n-1$ of the variables $\mathbf{x}=x_{1}, \ldots, x_{n}$. We shall let $\mathbf{n}$ denote the unit inner normal to the boundary $\partial \Omega$.

As usual $C^{k+\alpha}(\Omega)$ denotes the class of functions whose $k$ th order partial derivatives are Hölder continuous at exponent $\alpha, 0<\alpha \leq 1$. We shall be primarily 
interested in $C^{0+\alpha}(\Omega)$ and we will denote the usual norm on this space by $\|\cdots\|_{\alpha}$. Let $S$ denote the nonnegative cone of $C^{0+\alpha}(\Omega)$, that is

$$
S=\left\{h(x): h \in C^{0+\alpha}(\bar{\Omega}), h(x) \geq 0\right\} .
$$

By $C^{0}(\Omega)$ we mean the space of continuous functions on $\Omega$, and equip it with the supremum norm, $\|\cdots\|_{\infty}$.

We shall assume that

(A1) The undetermined coefficient $a(x) \in S$.

(A2) The initial data $f(x) \in C^{1+\alpha}(\Omega), f \geq 0$, and $\operatorname{grad} f=0$ on $\partial \Omega$.

For any $T>0$ it is well known [2] that the solution $u(x, t) \equiv u(x, t ; a)$ to the equations

$$
\begin{gathered}
u_{t}-\Delta u+a(x) u=0, \quad(x, t) \in \Omega \times(0, T), \\
\partial u / \partial \mathbf{n}=0, \quad(x, t) \in \partial \Omega \times(0, T), \\
u(x, 0)=f(x), \quad x \in \Omega,
\end{gathered}
$$

lies in $C^{2+\alpha}(\Omega)$ for each $t, 0<t<T$.

In order to determine both $a(x)$ and $u(x, t)$ we have to impose an additional boundary condition and as we stated in the introduction we shall require that

$$
u(x, T)=g(x)
$$

for some function $g(x)$. For the moment we shall assume only that $g(x) \in C^{0+\alpha}(\Omega)$, and without loss of generality assume that $T=1$. Our result is the following.

THEOREM. There is at most one solution pair $(a, u)$ to (2.1)-(2.4).

Proof. Consider the mapping $a \rightarrow \mathbf{T}(a)$ defined by

$$
\mathbf{T}(a)=a+\lambda(u(x, 1 ; a)-g)
$$

where $u(x, 1 ; a)$ denotes the solution of $(2.1)-(2.3)$ with the coefficient $a(x)$ and $\lambda>0$ is a constant whose value we are free to chose. If $\mathbf{T}$ has a fixed point $a \in$ $C^{0+\alpha}(\Omega)$, then from $(2.5)$ the function $u(x, 1 ; a)$ has to agree with $g(x)$. Conversely, if $g(x)=u(x, 1 ; a)$ for some coefficient $a \in C^{0+\alpha}(\Omega)$, then $a(x)$ must be a fixed point of the mapping $\mathbf{T}$.

We shall show that on the space $S, \mathbf{T}$ is a strict contraction and hence if it has a fixed point then that fixed point must be unique.

For any $a, h \in S$ we can easily compute the Gateaux derivative of $\mathbf{T}$ to obtain

$$
\mathbf{T}^{\prime}(a) \cdot h=h-\lambda \hat{u}(x, 1 ; a, h),
$$

where $\hat{u}(x, t ; a, h)$ is a solution of the nonhomogeneous initial-boundary value problem

$$
\begin{gathered}
\hat{u}_{t}-\Delta \hat{u}+a(x) \hat{u}=h(x) u(x, t ; a) \quad \text { in } \Omega \times(0,1), \\
\partial \hat{u} / \partial \mathbf{n}=0 \quad \text { on } \partial \Omega \times[0,1], \\
\hat{u}(x, 0)=0 \quad \text { in } \Omega .
\end{gathered}
$$

From the maximum principle applied to $u(x, t ; a)$ it follows from the nonnegativity of $f(x)$ and $a(x)$ that

$$
0 \leq u(x, t ; a) \leq \sup _{x \in \Omega} f(x)
$$


with regularity on the right-hand inequality if and only if $f=$ constant, and equality on the left if and only if $f=0$.

Since $h(x) \geq 0$, a further application of the maximum principle show that

$$
0 \leq \hat{u}(x, t ; a, h) \leq \phi(x, t)
$$

where $\phi(x, t)$ is the solution of the equation

$$
\phi_{t}-\Delta \phi=h(x)\|f\|_{\infty}
$$

with zero initial and zero Neumann boundary conditions. Also for any $t>0, x \in \Omega$, and any nontrivial $f(x), \hat{u}(x, t ; a, h)=0$ only if $h(x)$ is identically zero. Thus for any $\lambda>0$ the right-hand side of (2.6) is strictly less than $h(x)$ for all $x \in \Omega$. If $\lambda$ is now chosen sufficiently small (depending only on the function $f(x)$ and the domain $\Omega$ ) then it follows that

$$
\left\|\mathbf{T}^{\prime}(a) \cdot h\right\|_{\infty}=\|h-\hat{u}\|_{\infty}<\|h\|_{\infty}
$$

for any $a, h \in S$. Since the mapping $h \rightarrow \hat{u}(x, t ; a, h)$ is continuous in the topology of $C^{0+\alpha}(\Omega)$, it follows that for any $a, b \in S$

$$
\|\mathbf{T}(a)-\mathbf{T}(b)\|_{\infty}<\|a-b\|_{\infty}
$$

and the uniqueness of any fixed point of the mapping $\mathbf{T}$ then follows.

REMARK. An examination of the mapping $\mathbf{T}$ shows that its definition is based on the following properties:

If $a(x) \geq b(x)$ on $\Omega$ then from the maximum principle, $u(x, 1 ; a) \leq u(x, 1 ; b)$. Thus if one makes an initial guess $a_{0}(x)$ that is larger than an $a(x)$ with $u(x, 1 ; a)=$ $g$ then since $\lambda>0$

$$
\mathbf{T}\left(a_{0}\right)=a_{0}+\lambda\left(u\left(x, 1 ; a_{0}\right)-g\right) \leq a_{0}
$$

Also if $a_{0}(x)$ is equal to $a(x)$ except in the region $\Omega_{0} \subset \Omega$ then $u\left(x, 1 ; a_{0}\right)$ should differ from $u(x, 1 ; a)$ by a greater amount in the set $\Omega_{0}$ than in $\Omega-\Omega_{0}$.

Thus the term $\lambda(u(x, 1 ; a)-g)$ should be looked on as a compensating factor, with $\lambda$ a weight for the iteration scheme

$$
a_{n+1}=\mathbf{T}\left(a_{n}\right) \text {. }
$$

Implementing this iteration scheme on a computer for a wide range of coefficients $a(x)$ showed it to be quite robust and successful in finding a function $a(x)$ that makes $u(x, 1 ; a)$ arbitrarily close to the target $g(x)$.

If we could show that $\mathbf{T}$ mapped $S$, or one of its subsets, into itself, then by invoking the contraction mapping theorem we could obtain existence and continuous dependence of $a(x)$ on the functions $g(x)$ and $f(x)$ in the $C^{0+\alpha}$ norm (equation (2.13) can be extended to the $C^{0+\alpha}$ norm). The difficulty lies in identifying the correct conditions on the functions $g(x)$. If (A1) and (A2) hold then necessarily $u(x, 1 ; a)$ must lie in $C^{2+\alpha}(\Omega)$ and the same must be true of $g(x)$. Standard estimates would also give necessary bounds on the derivatives of $g(x)$, but showing the sufficiency of these estimates is another matter.

Perhaps one could obtain an existence result if the concept of solution to the undetermined coefficient problem was weakened in the following way:

We would say that the pair $(a(x), u(x, t))$ was a "generalized solution" to (2.1)(2.4) if there is a sequence of functions $a_{n}(x) \in C^{0+\alpha}(\Omega)$ converging to $a(x)$ in 
the $C^{0+\alpha}$ norm and such that the associated $u_{n}\left(x, 1 ; a_{n}\right)$ satisfying $(2.1)-(2.3)$ converged to $g(x)$ in the $C^{0+\alpha}$ norm. One could even replace convergence in $C^{0+\alpha}$ by sup norm convergence, and weaken the criterion of solution even further.

The above theorem gives the uniqueness of such a generalized solution. The convergence of the iterates of the scheme (2.14) would then give existence.

One could show the existence of a fixed point for $\mathbf{T}$ without being constrained to use the Contraction Mapping Theorem, but if this theorem could be invoked then the continuous dependence of $a(x)$ on $g(x)$ would follow. The following example will make it clear that one can only obtain a well-posed problem if the class of allowable coefficients is restricted to be smaller than $C^{0}(\Omega)$.

EXAMPLE. Let $\Omega=[0,1]$ and take $f(x)=1$. Choose $a_{n}(x)$ to satisfy

$$
a_{n}(x)= \begin{cases}0, & x \leq 1 / 2-1 / 2 n^{3} \\ 2 n^{3} x+n-n^{3}, & 1 / 2-1 / 2 n^{3}<x \leq 1 / 2 \\ -2 n^{3} x+n+n^{3}, & 1 / 2<x \leq 1 / 2+1 / 2 n^{3} \\ 0, & x \geq 1 / 2+1 / 2 n^{3}\end{cases}
$$

so that for $0 \leq x \leq 1, a_{n}(x)$ is a continuous nonnegative function whose $L^{1}[0,1]$ norm is $1 / n$. Thus $a_{n}(x) \rightarrow 0$ in $L^{1}$, but $\left\{a_{n}(x)\right\}$ fails to converge in the supremum norm.

As usual let $u^{(n)}(x, t)$ be the solution of

$$
\begin{gathered}
u_{t}^{(n)}-u_{x x}^{(n)}+a_{n}(x) u^{(n)}=0, \quad 0<x<1,0<t<1, \\
u_{x}^{(n)}(0, t)=u_{x}^{(n)}(1, t)=0, \quad 0 \leq t \leq 1 \\
u^{(n)}(x, 0)=1,
\end{gathered}
$$

and let $\psi(x, t)$ be the solution of the heat equation on $0<x<1,0<t<1$ with the boundary data (2.16), (2.17), so that $\psi(x, t) \equiv 1$ for all $x, t$. Then

$$
u^{(n)}(x, t)=\psi(x, t)+\int_{0}^{t} \int_{0}^{1} K(x, y, t-\tau) a_{n}(y) u^{(n)}(y, \tau) d y d \tau
$$

where $K(x, y, t)$ is the heat kernel corresponding to Neumann data on $x=0, x=1$. We have

$$
\begin{aligned}
u^{(n)}(x, t)-\psi(x, t)= & \int_{0}^{t} \int_{0}^{1} K(x, y, t-\tau) a_{n}(y) \psi(y, \tau) d y d \tau \\
& +\int_{0}^{t} \int_{0}^{1} K(x, y, t-\tau) a_{n}(y)\left\{u^{(n)}(y, \tau)-\psi(y, \tau)\right\} d y d \tau
\end{aligned}
$$

and Gronwall's inequality then gives

$$
\begin{aligned}
\sup _{0 \leq x \leq 1}\left|u^{(n)}(x, t)-\psi(x, t)\right| \leq & \left\{\int_{0}^{t} \int_{0}^{1} \sup _{0 \leq x \leq 1} K(x, y, t-\tau) a_{n}(y) \psi(y, \tau) d y d \tau\right\} \\
& \cdot \exp \left\{\int_{0}^{t} \int_{0}^{1} \sup _{0 \leq x \leq 1} K(x, y, t-\tau) a_{n}(y) d y d \tau\right\}
\end{aligned}
$$

Since $\int_{0}^{t} K(x, y, \tau) d \tau \leq C_{1}$ for some constant $C_{1}, 0 \leq x, y \leq 1$, and $\psi(x, t)=1$ we obtain

$$
\left\|u^{(n)}(x, 1)-1\right\|_{\infty} \leq C_{1}\left\{\int_{0}^{1} a_{n}(y) d y\right\} \exp \left\{C_{1} \int_{0}^{1} a_{n}(y) d y\right\} \leq \frac{C_{1}}{n} e^{C_{1} / n}
$$

that is, $u^{(n)}(x, 1) \rightarrow 1$ in the sup norm. 
Thus the inverse map which takes the target function $g(x)=u(x, 1)$ to the coefficient $a(x)$ is not continuous in the sup norm. We can actually show that the above $u^{(n)}(x, 1)$ converges to 1 in the $C^{1+\alpha}[0,1]$ norm for any $\alpha<1$ by modifying the estimates on the heat kernel $K$.

Notice that this example would fail if $a_{n}(x)$ were restricted to lie in a subset of $C^{0+\alpha}(\Omega)$ consisting of those functions whose $C^{0+\alpha}$ norm is uniformly bounded.

\section{REFERENCES}

1. J. R. Cannon, A class of inverse problems: The determination of second order elliptic partial differential operators from overspecified boundary data, Improperly Posed Problems, Pitman, San Francisco, Calif., 1975.

2. A. Friedman, Partial differential equations of parabolic type, Prentice-Hall, Englewood Cliffs, N.J., 1964.

3. I. M. Gel'fand and B. M. Levitan, On the determination of a differential equation from its spectral function, Amer. Math. Soc. Transl. (2) 1 (1955), 253-304.

4. A. Pierce, Unique determination of eigenvalues and coefficients in a parabolic problem, SIAM J. Control Optim. 17 (1979), 494-499.

5. W. Rundell, An inverse problem for a parabolic partial differential equation, Rocky Mountain J. Math. (to appear).

6. The determination of a coefficient in a parabolic differential equation from overspecified boundary data, Applicable Anal. (to appear).

7. T. Suzuki, Uniqueness and nonuniqueness in an inverse problem for the parabolic equation, J. Differential Equations 47 (1983), 296-316.

Department of Mathematics, Texas A\&M University, College Station, TEXAS 77843-3368 